\title{
El ser humano como conejillo de indias
}

\author{
Germán Novoa Heckel
}

\begin{abstract}
Resumen
La investigación clínica se relaciona con experimentos realizados en humanos; lo anterior ha sido legislado, de muchas maneras. A lo largo del camino recorrido en los últimos setenta años por la ética en la investigación clínica. Los humanos juegan aquí un papel fundamental que es fuertemente regulado. Es necesario conocer algunos pormenores, presentados a lo largo del texto, con el fin de que el paciente o voluntario pueda involucrase, con conocimiento de causa, en esta labor altruista orientada a desarrollar los medicamentos del mañana. Asimismo, el lector interesado podrá despejar algunas de las dudas más importantes relacionadas con la ética en la investigación clínica.
\end{abstract}

Palabras clave: investigación clínica, ética en investigación clínica, pacientes, medicamentos, protocolos de investigación.

\section{Humans as a gUINEA PIGS}

\begin{abstract}
Clinical research is related with experiments that are carried out in humans; this process has been legislated, in many ways, along the last seventy years by clinical research ethics. Humans play a fundamental role, a role that is heavily regulated. It is necessary to learn some of the details, presented along the text, with the goal of involving patients with sufficient knowledge and volunteers alike in this altruistic task oriented to the development of the drugs of tomorrow. The interested reader will also be able to clarify some of the most important questions related with clinical research ethics.
\end{abstract}

Key words: clinical research, ethics in clinical research, clinical research ethics, patients, drugs, research protocols. 


\section{Germán Novoa Heckel}

Especialista en Medicina Farmacéutica, maestro en Investigación Clínica, doctor en Investigación Médica, diplomado en Investigación Clínica, Administración, Mercadotecnia y Bioética, postdoctorante en el Programa Universitario de Bioética. Actualmente es Investigador en el Programa Universitario de Bioética de la UNAM.

\section{INTRODUCCIÓN}

El costo para llevar un solo medicamento al mercado se calcula entre 500 y 1000 millones de dólares. La industria multimillonaria de los fármacos desarrolla los nuevos productos que habrán de servir a la humanidad en los lustros que están por venir.

Existieron en un inicio abusos históricos, que culminaron con las atrocidades nazis durante la Segunda Guerra Mundial. Los humanos fueron tor-

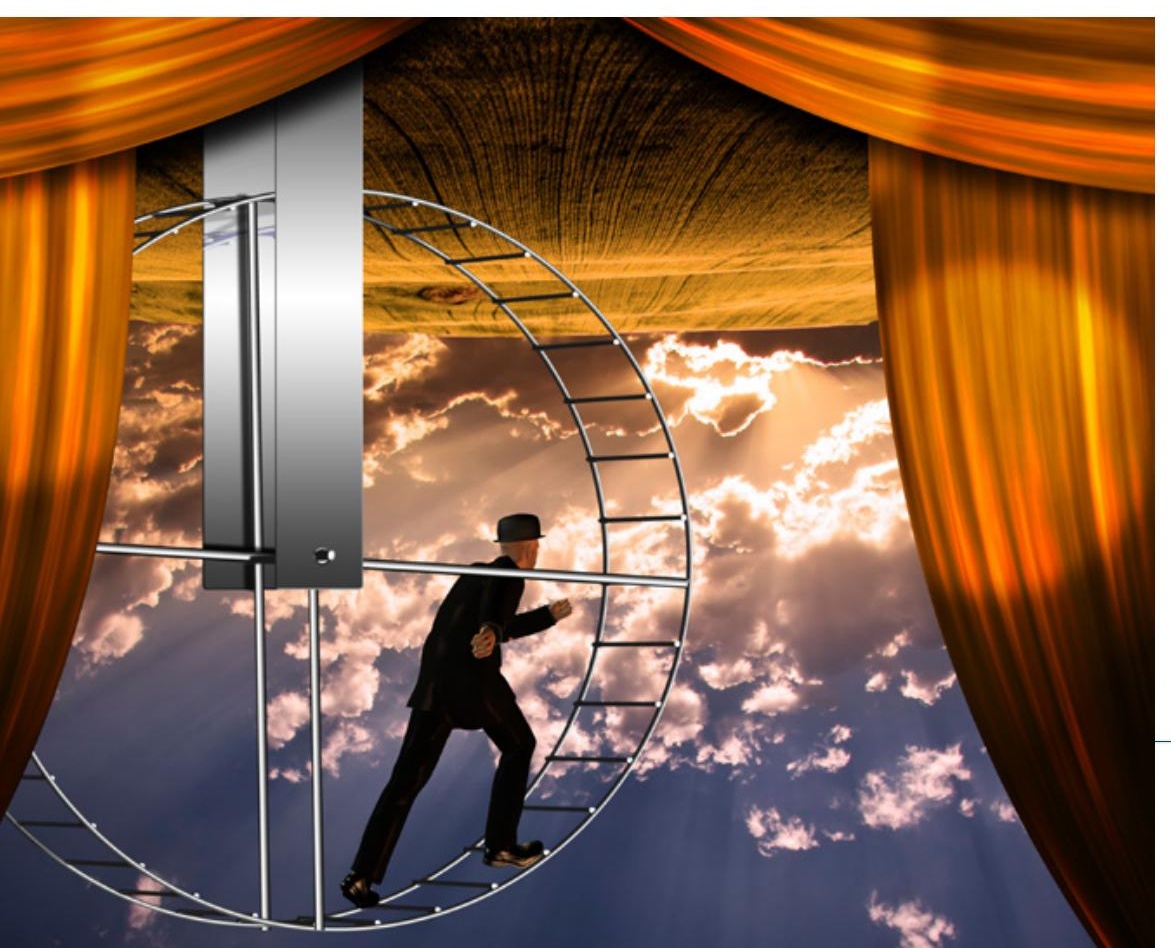
turados en ese entonces a partir de estudios con fines de investigación médica que no preguntaban a los sujetos, entre otros asuntos a considerar, si querían participar o no. Por otro lado, se investigaron asuntos en concreto que atentaron contra la integridad de las personas. Un ejemplo de lo anterior consistió en determinar la resistencia del ser humano al enfriamiento a partir de su inmersión en aguas heladas. El experimento tenía por objetivo descubrir métodos para mejorar la supervivencia de los soldados en sus campañas aéreas al cruzar los océanos sobre el mar. Después de la guerra, los experimentadores médi- 
cos fueron llevados a juicio y condenados por estas actividades atroces. Estos juicios (militares y médicos en su naturaleza) son conocidos como Procesos de Núremberg.

A partir de estas experiencias se fueron reglamentando las intervenciones permitidas en humanos con diversos códigos y reglamentos minuciosos que regulan, de manera estricta, la investigación clínica, que es la investigación con medicamentos, la que más frecuentemente solicita sujetos de estudio (humanos) para sus investigaciones. ¿Cómo se realiza la investigación en humanos y qué principios subyacen en ella para que sea ética y pueda responder a su llamado? ¿Qué necesitaría saber en caso de que fuera invitado a participar en alguna de estas investigaciones, ya sea como voluntario sano o como paciente, en alguno de los cientos y miles de estudios que se realizan simultáneamente en México y en muchos otros países del mundo, desde hace ya muchas décadas de investigación farmacéutica?

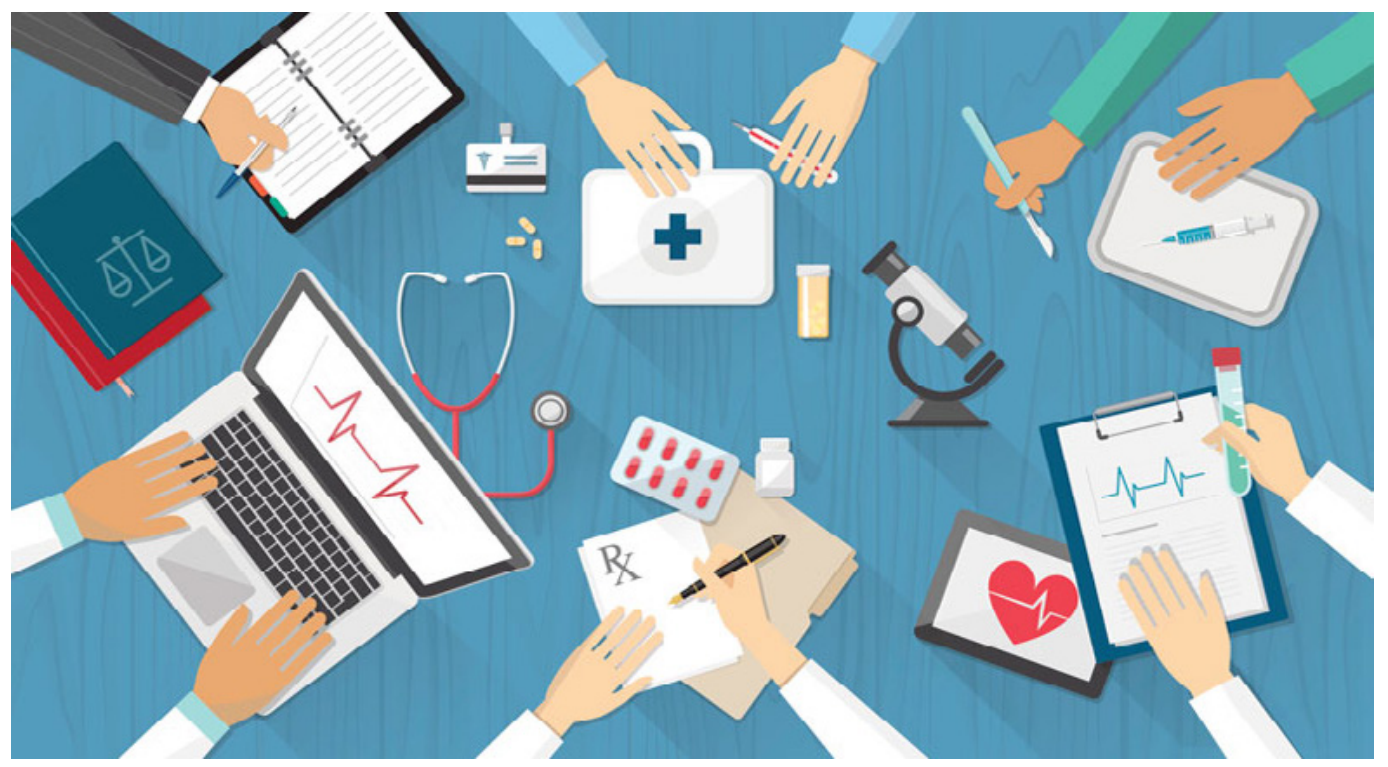

\section{La investigación clínica en pocas palabras}

Los laboratorios farmacéuticos producen y descubren ellos solos la gran mayoría de los nuevos medicamentos que aparecen anualmente en el mundo. Estos desarrollos dependen de la investigación clínica, que consiste en una compleja y regulada cadena de eventos que culminan con un medicamento nuevo. Lo anterior se realiza mediante una prueba clínica, bajo condiciones experimentales, de ciertas moléculas en los seres humanos. Este paso es ineludible, puesto que se tiene que iniciar la investigación del comportamiento del medicamento en el organismo del hombre por primera vez, y más vale que este estudio sea en condiciones controladas. Lo anterior sucederá, entonces, bajo estrictas normas 
de experimentación. Estos experimentos se pueden llevar a cabo a través de las Ilamadas Organizaciones de Investigación Clínica por Contrato o CROs (por sus siglas en inglés), quienes se dedican a la investigación clínica de manera exclusiva y tienen representantes en muchos países a nivel internacional. Así, las empresas farmacéuticas creadoras del nuevo medicamento contratan a dichos grupos de investigación (equipos que en su origen existían únicamente dentro del propio laboratorio farmacéutico, con equipos de investigación internos) y que estudian los medicamentos durante las fases I a IV del desarrollo farmacéutico en humanos. La fase I se realiza en voluntarios sanos para determinar su comportamiento en el organismo (farmacología del medicamento) en unos pocos voluntarios sanos (aproximadamente 5-10 voluntarios) y el objetivo es probar la seguridad; en la fase II se prueba el fármaco por primera vez en enfermos, y se determina su utilidad para la patología para la que fue creado, con lo cual el objetivo es evaluar su eficacia y seguridad; la fase III se lleva a cabo en una población de enfermos más amplia (algunos cientos a pocos miles de

La investigación deberá ser llevada a cabo de acuerdo con principios éticos universales

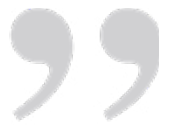
participantes) para ver la seguridad - a partir de la incidencia de efectos adversos-, y se varían algunas condiciones, como por ejemplo la concomitancia con otros medicamentos y las condiciones bajo cierta alimentación; finalmente, en la fase IV, con el medicamento ya comercializado y en el mercado, se estudia en poblaciones abiertas su comportamiento para corroborar —como objetivo - la seguridad en cientos y miles de pacientes, para determinar si hay efectos secundarios raros que se puedan presentar en pocos individuos (un efecto en 10,000 pacientes o un efecto en 100,000 pacientes), los cuales son más difíciles de detectar en las fases previas, con apenas unos cientos o pocos miles de sujetos de investigación.

De diez mil moléculas investigadas, se conoce que entre dos y tres llegan a las etapas de investigación clínica de fases I-III y sólo una finalmente al mercado, una vez demostrada su eficacia y su seguridad ante las autoridades pertinentes. En México es la Comisión Federal para la Protección contra Riesgos Sanitarios (COFEPRIS) la agencia gubernamental encargada de aprobar o rechazar medicamentos. Estas aprobaciones se dan a partir de la evaluación de cientos de miles de datos recogidos durante las arduas investigaciones realizadas durante muchos años, en donde el medicamento llega a su última etapa de registro, que culminará con los permisos para su venta. En cuanto a la ética de estas investigaciones, se ha dicho que "La sociedad justamente espera que la investigación médica en sujetos humanos esté supervisada con rigor. Uno de los aspectos es asegurar que la investigación sea ética. La otra faceta es que exista revisión experta del trabajo 
por realizarse. Los investigadores deben mostrar a otros, sin lugar a duda, que la investigación es adecuada en todo sentido" ([Editorial], 1997). Lo anterior resume los aspectos éticos fundamentales de la investigación clínica en humanos.

Los pacientes que participan en estas investigaciones son la parte medular del desarrollo de los medicamentos, y son seleccionados siguiendo reglamentos y pautas éticas específicas y detalladas, y principios que subyacen en toda investigación con humanos en condiciones experimentales. Dicha reglamentación ha llegado hasta nuestros días ya muy perfeccionada, con códigos de comportamiento y de actuación puntuales reconocidos mundialmente, como la Declaración de Helsinki, así como la Declaración del Consejo de Organizaciones Internacionales de las Ciencias Médicas (CIOMS, por sus siglas en inglés), el Código de Regulaciones Federales (CFR por sus siglas en inglés) de los Estados Unidos y las Guías de la Conferencia Internacional de Armonización (ICH por sus siglas en inglés), que no sólo regulan la participación de humanos, en todos sus aspectos, sino que, adicionalmente, fijan las reglas y establecen los pormenores de cada una de las actividades y de la logística implicadas en los experimentos realizados con medicamentos en cada una de las fases.

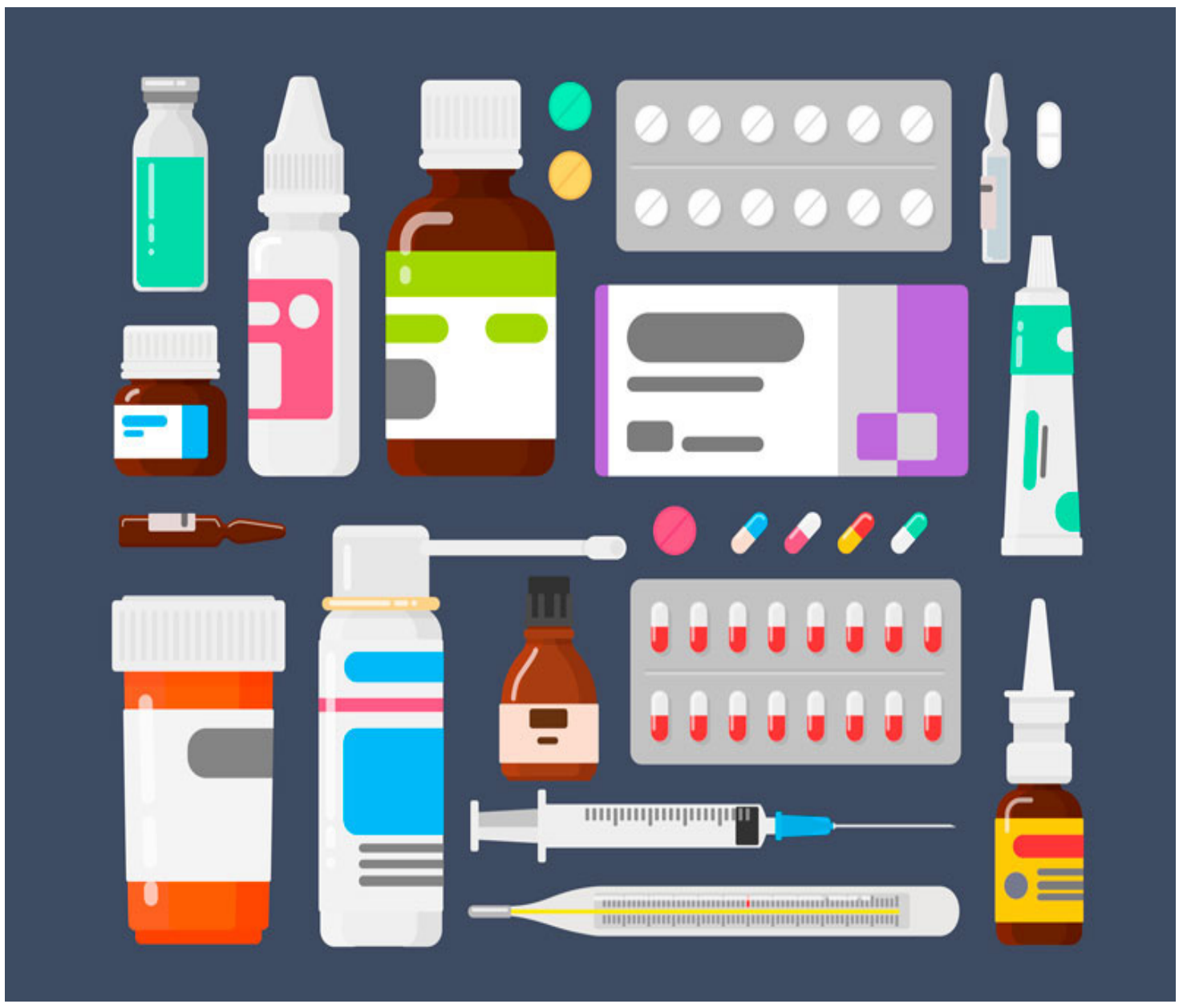




\section{Principios de ética necesarios en las investigaciones con humanos}

Entonces, cabe preguntarse, ¿cuáles son estos principios fundamentales que regulan la participación de humanos en investigación clínica? (ver tabla 1). Se considera que una investigación es ética con referencia al diseño y desarrollo de la investigación en sí misma, cuando cumple con siete requisitos, planteados originalmente por Emanuel (2000), y publicados a principios del milenio (2000) en la prestigiosa revista Journal of the American Medical Association (JAMA).

\begin{tabular}{|ll|}
\hline $\begin{array}{r}\text { Tabla 1. Siete requisitos para que una } \\
\text { investigación en humanos sea ética } \\
\text { (Emanuel, E. J. et al., 2000) }\end{array}$ \\
\hline 1. & Valor (value) \\
\hline 2. & Validez científica \\
\hline 3. & Selección con justicia de los participantes \\
\hline 4. & Balance favorable de riesgo/beneficio \\
\hline 5. & Revisión independiente \\
\hline 6. & Consentimiento informado \\
\hline 7. & Respeto hacia los participantes \\
\hline
\end{tabular}

El valor se refiere a la importancia social, científica o clínica: el estudio deberá conducir a mejoras en la salud, conducir al bienestar de la población, y generar información de los sistemas biológicos. Es un requisito ético por dos razones: por el uso responsable de recursos limitados y porque se debe evitar la explotación, es decir, no exponer a riesgos sin la posibilidad de beneficio.

La validez científica estipula que la metodología debe ser válida y realizable: con un objetivo científico claro; principios, métodos y prácticas seguros y aceptados; con un poder suficiente para probar el objetivo; con un plan de análisis de datos verosímil, que debe poder llevarse a cabo.

La selección con justicia de los participantes se refiere a los grupos de sujetos relacionados con el estudio, los cuales deberán tener todos ellos la misma oportunidad de participar.

El balance favorable de riesgo-beneficio implica que los riesgos potenciales se minimizan y los beneficios potenciales a los sujetos individuales o a la sociedad se maximizan. Significa también que los beneficios potenciales son proporcionales o exceden los riesgos asumidos y que los sujetos estarán en condiciones de beneficiarse con un resultado positivo. 
En cuanto a la necesidad de una revisión independiente, la misma se realiza por los llamados comités de ética en investigación, grupos especialmente constituidos para una revisión ética experta de la propuesta de investigación.

La base esencial de la colaboración por parte de sujetos de estudio participantes está dada por un documento denominado "consentimiento informado" que existe para considerar una participación auténticamente voluntaria, a partir del hecho de que se ha comprendido la esencia misma de la investigación y que se le ha explicado al sujeto en cuestión, de forma pormenorizada, cada una de las actividades que se realizarán durante el experimento. Adicionalmente, el sujeto puede preguntar y aclarar toda duda que hubiera surgido de la lectura detenida del documento (que sigue pautas muy específicas en su elaboración), para así respetar la autonomía de las personas (que consiste - esta autonomía- en el hecho de que todos tenemos la libertad de decidir y expresar nuestro sentir a nuestra mejor conveniencia, sin la intervención de terceros que pretendan influir sobre nuestras decisiones, libres de toda explotación o abuso) e incluye toda la información por escrito que debe conocer el sujeto antes de colaborar. El documento incluye, asimismo, la garantía de que el participante podrá retirarse en el momento que así lo desee sin ningún tipo de represalias. El participante consigna entonces que ha comprendido y que ha podido hacer todas las preguntas que le hayan surgido, y que éstas han sido respondidas satisfactoriamente. Al final, y como señal de que se aceptan las condiciones estipuladas, se firma el documento ante dos testigos, y una copia del documento se le entrega al voluntario.

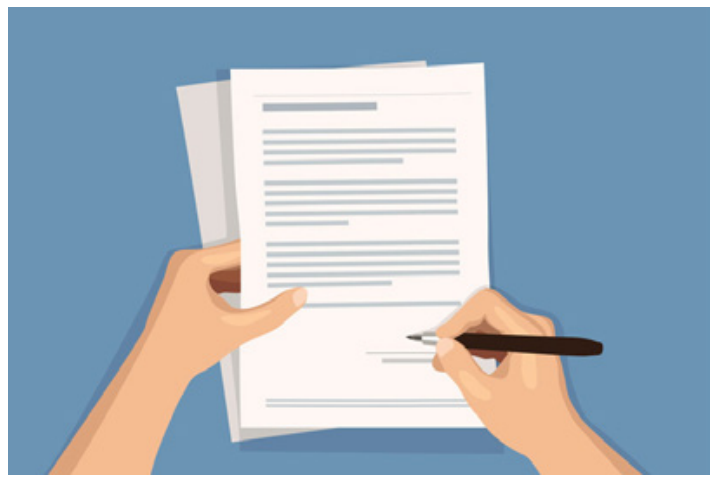

Se entiende aquí que no es fácil obtener un consentimiento totalmente claro de los voluntarios, ya que los grados de entendimiento siempre varían, dependiendo de la educación y cultura médica que tenga cada firmante. El documento constituye, como ya se ha expresado, la esencia y el requisito ineludible de la participación ética de los voluntarios en un estudio clínico. Su participación queda documentada como un acto de voluntad manifiesta y legalizada, sin ser lo legal el factor único del pacto.

El respeto hacia los participantes implica permitir al sujeto cambiar de opinión y retirarse; respetar su privacidad (reglas de confidencialidad); informar los datos nuevos acerca de riesgos y beneficios; informar a los sujetos los resultados de la investigación y finalmente, vigilar el bienestar del sujeto a lo largo de su participación.

El individuo participante se someterá, una vez aceptada su participación, a una serie de actividades descritas en el protocolo de estudio, que típicamente 
incluye interrogatorios y estudios clínicos y de gabinete, además de diversos procedimientos que variarán para cada tipo de estudio del que se trate (no será lo mismo un estudio cardiovascular que, por ejemplo, una investigación neurológica o dermatológica).

El participante acudirá a sucesivas visitas de control y seguimiento, e ingerirá el medicamento de estudio bajo el régimen solicitado, a lo largo del tiempo en que se lleve a cabo el experimento, siempre bajo la mirada atenta y solícita del equipo de investigadores.

\section{¿Cómo puedo contribuir como voluntario en una investi- gación de un medicamento nuevo?}

¿Qué obligaciones y requisitos hay para participar en un estudio de estas características? En un principio, el requisito es el de aceptar participar. La cooperación de las personas está basada en el hecho de querer contribuir, altruistamente, con el conocimiento que surgirá de las investigaciones (los resultados del estudio) y que será de utilidad en un futuro a otros pacientes en diversas partes del planeta. Esta ayuda se basa en el compromiso social que ofrecemos como miembros de una sociedad organizada; contribuimos, libre y desinteresadamente, para que otros se beneficien de los resultados en un futuro. La participación colaborativa llena aquí un hueco importante que debe resaltarse con mucho énfasis. A continuación, tendremos que aceptar las obligaciones descritas en el documento de consentimiento informado, a las que nos comprometemos al firmar el mismo.

Adicionalmente, habría que aclarar aquí que, por lo general, no siempre habrá un beneficio de tipo terapéutico, lo cual tiene que ser informado al sujeto prospectivo en el consentimiento informado. Lo anterior es causa frecuente de confusión entre los voluntarios, que anticipan tal vez la cura de sus padecimientos y la eliminación de todos sus malestares; por lo anterior, se deberá aclarar este hecho al paciente y concientizar a la población, siempre que sea posible, con el fin de no generar falsas expectativas. No siempre habrá un beneficio.

La participación, en algunos casos (sobre todo en los estudios de voluntarios sanos), podría conllevar una compensación económica. Las investigaciones en las que participan voluntarios sanos son aquellas en las que se prueban medicamentos que requieren aprobación oficial como genéricos, en estudios llamados de bioequivalencia. Se determina aquí que los productos originales y las copias — genéricos - son lo más parecidas posibles. En otros tipos de estudios, en este caso los estudios sobre enfermos, usualmente, y con más frecuencia, a los pacientes se les pueden pagar los viáticos, de transporte o alimentos, aunque no en todos los casos. Los servicios proporcionados durante el estudio (consultas, medicamentos, estudios de laboratorio y gabinete), sin embargo, siempre serán gratuitos. 


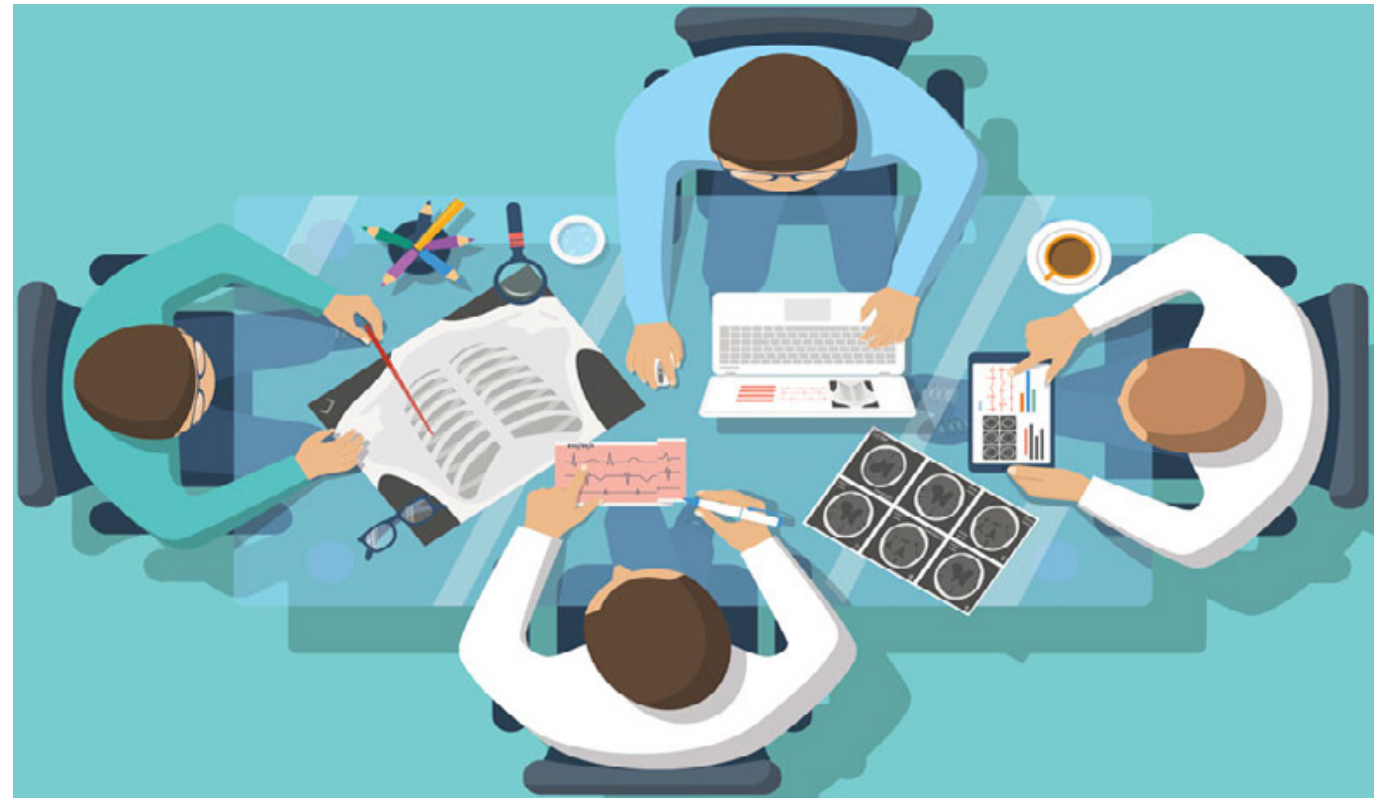

Otro asunto importante: la investigación se realiza considerando riesgos razonables y un balance riesgo-beneficio tolerable. Antes de iniciar una investigación, el protocolo y los datos del medicamento estudiado, así como las credenciales del investigador y de sus asistentes, entre otros, son sometidos a escrutinio por parte de los comités de ética de la investigación que revisan la propuesta de estudio y determinan la magnitud de los riesgos a partir de un balance riesgo-beneficio. De igual manera, evalúan el potencial que tiene el estudio de algún abuso, conocido esto genéricamente como riesgo de explotación o abuso (hablaremos de esto más adelante en detalle), el cual es más probable que se presente cuando se trata de poblaciones vulnerables (la vulnerabilidad puede ser económica, cultural, social, entre otras). El comité de ética en investigación deberá corroborar la idoneidad en la adherencia a principios éticos, que incluyen la no existencia de abuso. Los principios son determinados por instancias internacionales, y revisados continuamente.

Dentro de las actividades del comité de investigación, que es diferente de la del comité de ética en investigación, está la de revisar la propuesta desde el punto de vista metodológico; se valora la conformación procedimental, y sus detalles técnicos y científicos, que deben ser apropiados, suficientes y congruentes. Ambas comisiones (ética e investigación) evalúan el proyecto en su conjunto, recomiendan modificaciones en caso de ser requeridas y aprueban o no, en última instancia, la investigación. Posteriormente, los documentos aprobados se turnan a la autoridad gubernamental para su visto bueno final. En México dicha instancia es la COFEPRIS, como ya se mencionó al inicio de nuestra exposición. 
En el consentimiento informado (que forma parte de los documentos que se revisan) se consignan los datos de contacto del comité y de los investigadores. La accesibilidad de estos datos posibilita una consulta directa al investigador, si es que así lo desea el participante. Puede ser que quiera aclarar asuntos que surjan con posterioridad. La participación en los estudios se da por invitación de los médicos tratantes, a los cuales hemos acudido para una consulta, o por invitación directa de los investigadores y sus equipos (menos frecuente), por avisos en tableros en clínicas y hospitales, publicidad en medios especializados, aunque también, en ocasiones, es el propio paciente, a partir de comentarios de familiares o amigos, el que busca algún centro de investigación para colaborar. En el caso de los voluntarios sanos para estudios de bioequivalencia, los mismos son convocados por anuncios y publicidad expresa para este tipo de estudio.

\section{El estudio propiamente dicho}

Una vez cumplidos los requisitos necesarios para participar (un perfil específico en casi todos los casos) - por ejemplo, que el participante padezca diabetes de reciente diagnóstico o de evolución de un determinado número de años, o presencia de otro padecimiento que pudiera ser objeto del estudio hipotético, como asma, alguna enfermedad del grupo de las infecciones u otra situación aguda o crónica - el voluntario es incluido para la investigación. Otros requerimientos exigen, en ocasiones, cierto género, edad o particularidades específicas de la enfermedad; que no se presenten al mismo tiempo ciertos padecimientos (llamadas concomitancias, no permitidas entre otras razones, porque se ingieran otros medicamentos y porque enmascaren la investigación pretendida) y otros aspectos restrictivos consignados como criterios de inclusión y exclusión del protocolo de estudio. Lo anterior con el fin de restringir las variables de la experimentación, para evaluar e interpretar adecuada y objetivamente los resultados. Mientras menos variables haya que valorar, más objetiva será la evaluación final.

Es fundamental que el participante tenga disponibilidad de tiempo para acudir a las visitas, en donde en muchas ocasiones sólo se requiere que se presente al interrogatorio médico (una vez realizadas las pruebas de laboratorio y gabinete iniciales rutinarias); también es posible que se pidan requisitos adicionales, como, por ejemplo, llenar un diario o utilizar artefactos electrónicos de seguimiento - por ejemplo, un Holter de monitoreo cardiaco, que es un dispositivo que valora el funcionamiento del corazón- proporcionados al paciente y libres de costo.

La duración del estudio podrá variar ampliamente, y puede ir de pocas semanas (una o dos), hasta varios meses (lo más frecuente entre tres y seis) o años (entre uno y máximo de dos normalmente), dependiendo de la naturaleza de cada investigación. Estudios como el asma requieren valoraciones de más tiempo, ya que los episodios de agudización pueden ser muy espaciados. 
Las ventajas de participar (quisiéramos desde luego encontrar alguna, cuando ya hemos dicho que no siempre hay un beneficio terapéutico), generalmente las encontramos en el seguimiento minucioso que se realiza de la propia enfermedad y del estado de salud en general. Hay frecuentemente aprendizajes importantes sobre la propia patología para el sujeto (por ejemplo, en estudios de diabetes, o reumatología, que conllevan educación importante para el participante y su enfermedad). Otras ventajas consisten en que se adopta una disciplina de tratamiento rigurosa que nos permite aprender a cumplir mejor con nuestro tratamiento, principalmente en enfermedades crónicas. Otras ventajas, finalmente, consisten en entender y aprender acerca de la ciencia y sus métodos.

Entre las desventajas, están el tiempo que se tiene que dedicar, la necesidad de apego a los protocolos que exigen cierto grado de disciplina y, a veces, algunas incomodidades dadas por las pruebas o los tratamientos. Todo esto influye sobre nuestra rutina y nuestro estilo de vida habituales; por ejemplo, ocasionalmente la necesidad de cumplimiento de un régimen dietético, o de una visita programada o extraordinaria, así como de la toma de medicamento en horarios precisos y de forma determinada (con cierta cantidad de agua o en circunstancias específicas como durante una exacerbación del padecimiento). Si no se tiene esta disposición mínima de cooperación y de búsqueda de cumplimiento con el comportamiento deseado, tal vez sea recomendable el no participar en estudios de investigación.

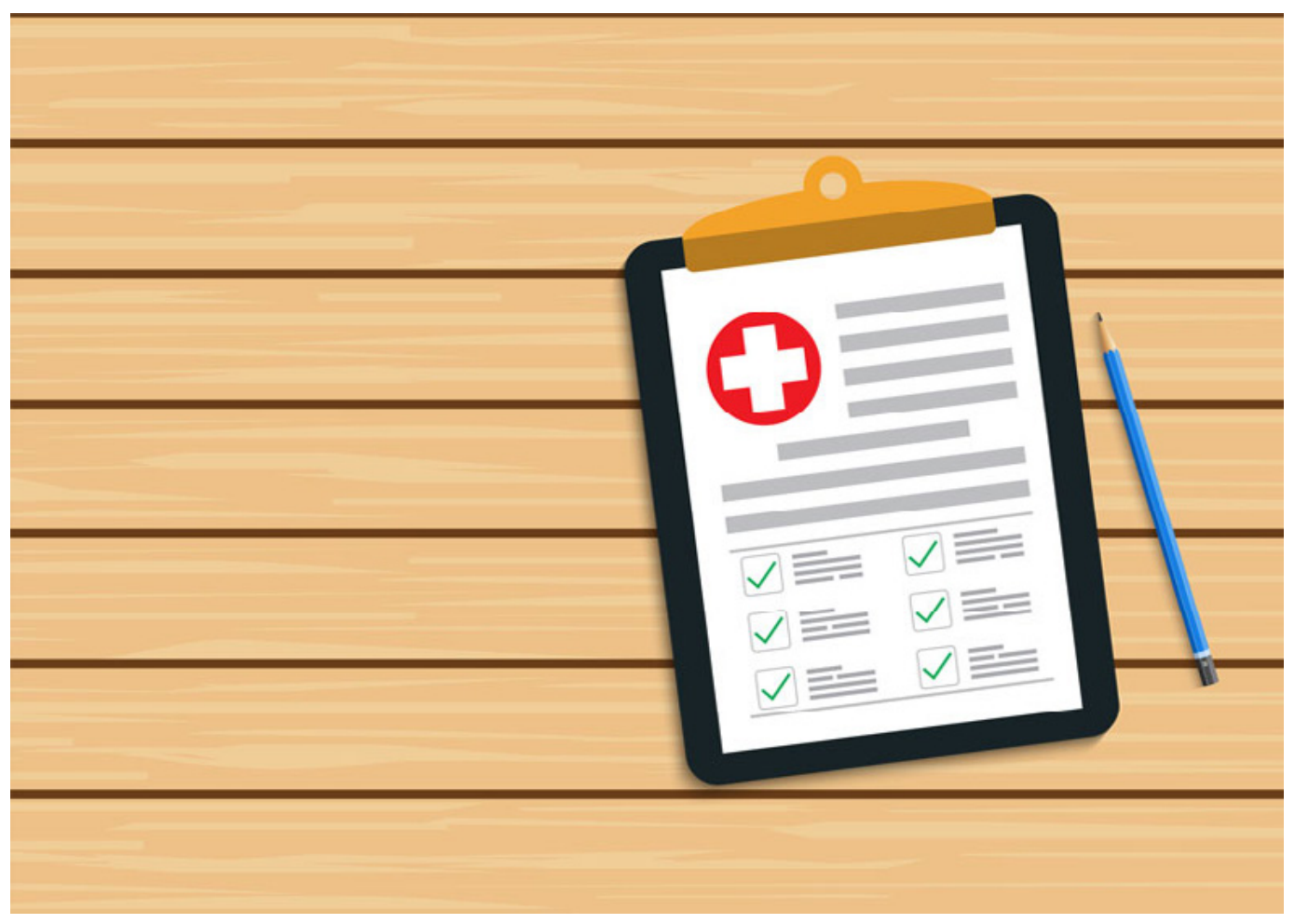




\section{Principios éticos en la investigación en humanos}

La investigación debe llevarse a cabo, decíamos, de acuerdo con principios éticos universales. Aunque estos principios básicos fueron violados con frecuencia antes de la creación del primer código protector, el Código de Núremberg, hoy en día se han ampliado mucho, y son muchos los códigos existentes (ya mencionados), además de otros muchos reglamentos nacionales e internacionales más recientes (como los de la Unión Europea), que protegen a los sujetos de experimentación en todo momento, y son observados en su gran mayoría, mismos que regulan minuciosamente la participación de sujetos en estudios de investigación clínica.

En una de las definiciones conocidas de la palabra ética, para adentrarnos un poco en este asunto fundamental de la investigación, encontramos que la misma es la aplicación de los valores y de las reglas morales a actividades humanas. En nuestro caso, esta aplicación de valores morales busca encontrar soluciones razonadas, consistentes y defendibles a los dilemas de participación humana en investigaciones. La bioética, tan de moda actualmente, es una parte de esa ciencia ética, y utiliza los principios éticos para tomar decisiones y resolver dilemas existentes o anticipados en la medicina y también en la biología y en relación con el medio ambiente. Ahora bien, el principialismo, una postura ética surgida justamente a partir de la investigación en humanos, es la corriente de la ética universal, propuesta por dos autores norteamericanos: Tom Beauchamp y James Childress (2001), que se ocupa, más precisamente, de los dilemas de la investigación clínica y biomédica.

Los principios éticos que se deben considerar en toda investigación (aplicables también en otros análisis éticos), de acuerdo con el principialismo, son cuatro:

- Autonomía (participación libre sin coerción).

- Beneficencia (anticipación de algún beneficio).

- No maleficencia (ausencia de daño).

- Justicia (deber o no de participar en un estudio).

La autonomía de los individuos es la capacidad y libertad de una persona de tomar decisiones personales. En particular es ejercido mediante el consentimiento libre e informado, que, como se mencionó al principio, puede ser retirado en el momento que así se decida. Es el principio fundamental de la ética en la investigación con humanos y está basado en el hecho de que es la decisión más importante que podemos tomar en relación a que se experimente en nosotros: los sujetos en los campos de concentración durante la segunda guerra mundial no tuvieron esa libertad.

La beneficencia y la no maleficencia requieren maximizar lo primero (el beneficio) y minimizar lo segundo (la maleficencia o daño). En particular, se cumplen a partir de un diseño sólido que siga criterios de calidad científica. También 
se refuerza a partir del hecho de que los investigadores sean competentes y cumplan con las obligaciones y los estándares profesionales relevantes. La no maleficencia inicia con el precepto médico del primum non nocere, locución latina que significa que lo primero como médico, y ante un paciente, es no hacer daño.

La justicia engloba la imparcialidad y la equidad. Cumple con el mandato de quién debería beneficiarse de la investigación y soportar los riesgos y cargas que conlleva. Este principio es conocido como justicia distributiva, y comprende la elección (con justicia) de los candidatos que participarán en la investigación.

Los requisitos (tabla 1) para que una investigación en humanos sea ética ya fueron consignados al inicio de este escrito, y no se comentarán más.

Finalmente, es importante mencionar el riesgo que tienen las poblaciones de estudio de una posible explotación. La explotación se refiere al hecho de que se puedan cometer abusos (abuso de la buena voluntad de los sujetos, de su tiempo, de su comodidad, de su paciencia, entre otros) a los individuos durante la selección y realización del estudio. Es por esto por lo que se tiene que insistir en los preceptos éticos de protección a los pacientes. Lo anterior es tomado con mucha seriedad hoy en día, y la posibilidad de abusos en investigación clínica ha disminuido de forma sustancial.

Mencionaremos también, para fines didácticos, que el riesgo de explotación es aún mayor cuando las condiciones de una población son deficientes, como es el caso frecuentemente en las sociedades de latinoamerica, entre otras a nivel mundial. Las condiciones adversas que prevalecen en nuestras poblaciones han sido reseñadas con anterioridad (Campos y Cabral, 2015), y aquí mostramos un breve listado de asuntos de vulnerabilidad, que contribuyen al riesgo de explotación (tabla 2). Este tipo de explotación puede y debe ser tomado en cuenta para prevenirlo a través de la revisión ética concienzuda de los comités de ética en investigación.

Tabla 2. Condiciones sociales, culturales y económicas de marginación en poblaciones, que contribuyen al riesgo de explotación (Campos y Cabral, 2015)

\begin{tabular}{|l|}
\hline Pobreza \\
\hline Insuficiente educación general y de oferta \\
\hline Pobreza por distribución desigual de la riqueza \\
\hline Desorganización y falta de calidad en la atención pública de la salud \\
\hline Dificultad en el acceso a la salud \\
\hline Costumbres culturales y religiosas \\
\hline Desigualdades sociales de clase y género \\
\hline Estructuras del poder \\
\hline Liderazgo de sujetos e investigadores con conflicto de interés \\
\hline
\end{tabular}




\section{Conclusiones}

La investigación en humanos se ha realizado de forma organizada y regulada a lo largo de más de setenta años a nivel internacional, con el fin de llevar al mercado los medicamentos que urgentemente requiere la humanidad. Estamos lejos todavía de poder curar la mayoría de las enfermedades. La ética de estas investigaciones ha sido fundamental para construir la infraestructura necesaria que ha aportado a todos medicamentos indispensables, de los cuales hoy en día ya no podemos prescindir. Entre ellos contamos los antiinfecciosos, antiinflamatorios y analgésicos, pero también los hipoglucemiantes, los cardiovasculares y los oncológicos, por nombrar solamente unos cuantos de los grandes grupos terapéuticos existentes. Los principios éticos incluidos representan un amplio abanico de valores, importantes en toda investigación que contemple la inclusión de humanos, aún más allá de los estudios con medicamentos aquí reseñados.

El participante en investigación clínica informado, con múltiples sitios posibles de revisión documental especializada, tiene siempre la mejor opción para cumplir a cabalidad con su cometido altruista de colaboración aquí esbozado, en donde será importante saber tanto lo que puede esperar como lo que puede exigir. El conocimiento así adquirido le permitirá entender también la relevancia de su participación, y la importancia e implicaciones de firmar un consentimiento informado, signando con conocimiento de causa completo.

Sería fundamental el conocer más acerca de los códigos y regulaciones en la materia si se quiere entender mejor el espíritu ético y humanista que guía estas investigaciones, pudiendo partir en un inicio de las menciones que se hacen a lo largo de este texto. La ética en la investigación clínica constituye un firme pilar de la ética internacional y es una muestra fehaciente de los resultados espectaculares que se pueden alcanzar cuando la comunidad, en este caso la de la investigación, se pone de acuerdo para dictaminar pautas de comportamiento de naturaleza universal deseadas. Los diferentes códigos y pautas existentes son testimonio de lo anterior, y están disponibles para ser analizados y valorados por quien así lo desee.

\section{Referencias}

Beauchamp, T. L. y Childress, J. F. (2001). Principles of Biomedical Ethics [5th ed.]. Nueva York: Oxford University Press.

[Editorial]. (1997). International Journal of Pharmaceutical Sciences and Research, $\|, 3: 1194$.

* Emanuel, E. J., Wendler, D. y Grady, C. (2000). What makes Clinical Research Ethical? Journal of the American Medical Association (JAMA). 28(20), 2701-11. 
* Emanuel, E., Grady, C. y Crouch, R. (2008). The Oxford Textbook of Clinical Research Ethics. Nueva York: Oxford University Press.

* Campos Pavone, E. L. y Cabral Schveitzer, M. (2015). Responsabilidad Social de los Investigadores. En Álvarez Díaz, J. A. y López Moreno, S. (Eds.), Ensayos sobre Ética de la Salud. Investigación. México: Universidad Autónoma Metropolitana. pp. $31-42$.

* Secretaria de Salud (2016). Ley General de Salud. Recuperado de: http://www. salud.gob.mx/cnts/pdfs/LEY GENERAL DE SALUD.pdf. [Consultado: 23 de septiembre, 2013].

* Secretaria de Salud (1986). Reglamento de la Ley General de Salud en Materia de Investigación para la Salud. Recuperado de: http://www.salud.gob.mx/unidades/ cdi/nom/compi/rlgsmis.html

* Secretaria de Gobernación (2013, 4 de enero). NORMA Oficial Mexicana NOM-012SSA3-2012, Que establece los criterios para la ejecución de proyectos de investigación para la salud en seres humanos. Ciudad de México: Diario Oficial de la Federación. Recuperado de: http://dof.gob.mx/nota detalle.php?codigo=528414 8\&fecha $=04 / 01 / 2013$

* Secretaria de Gobernación (2012, 31 de octubre). ACUERDO por el que se emite las Disposiciones Generales para la Integración y Funcionamiento de los Comités de Ética en Investigación y se establecen las unidades hospitalarias que deben contar con ellos, de conformidad con los criterios establecidos por la Comisión Nacional de Bioética. Ciudad de México: Diario Oficial de la Federación. Recuperado de: http://www.dof.gob.mx/nota detalle.php?codigo=5276107\&fec $\underline{h a}=31 / 10 / 2012$

* National Institutes of Health (2018). Aprenda más sobre investigación clínica de los NIH. Recuperado de: https://salud.nih.gov/investigacion-clinical

- Lilly (2018). Los pacientes y la Investigación Clínica. Recuperado de: http://www.lilly. es/es/investigacion-y-desarrollo/la-investigacion-clinica/los-pacientes-y-la-investigacion-clinica.aspx

- Canifarma (2017, 7 de septiembre). Investigación clínica: ¿Qué debo saber antes de participar? Recuperado de: http://codigof.mx/investigacion-clinica-base-para-la-innovacion-ii-partel.

\section{Cómo citar este artículo}

- Novoa Heckel, Germán (2018). El ser humano como conejillo de indias. Revista Digital Universitaria (RDU). Vol. 19, núm. 4 julio-agosto. DOI: http://doi.org/10.22201/ codeic.16076079e.2018.v19n4.a2. 